\title{
The newly synthesized 2-(3-hydroxy-5-methoxyphenyl)-6,7- methylenedioxyquinolin-4-one triggers cell apoptosis through induction of oxidative stress and upregulation of the p38 MAPK signaling pathway in HL-60 human leukemia cells
}

\author{
YUNG-YI CHENG $^{1}$, JAI-SING YANG ${ }^{2}$, SHIH-CHANG TSAI ${ }^{3}$, CHIH-CHUANG LIAW $^{5}$, \\ JING-GUNG CHUNG ${ }^{2,6}$, LI-JIAU HUANG ${ }^{1}$, KUO-HSIUNG LEE ${ }^{7,8}$, CHI-CHENG LU ${ }^{9}$, \\ HSI-CHENG CHIEN $^{3}$, MINORU TSUZUKI ${ }^{4,10}$ and SHENG-CHU KUO ${ }^{1,8}$
}

\begin{abstract}
${ }^{1}$ Graduate Institute of Pharmaceutical Chemistry, Departments of ${ }^{2}$ Pharmacology, and ${ }^{3}$ Biological Science and Technology,
${ }^{4}$ Tsuzuki Institute for Traditional Medicine, China Medical University, Taichung 404; ${ }^{5}$ Department of Marine Biotechnology and Resource, National Sun Yat-Sen University, Kaohsiung 804; ${ }^{6}$ Department of Biotechnology, Asia University, Taichung 413, Taiwan, R.O.C.; ${ }^{7}$ Natural Products Research Laboratories, Eshelman School of Pharmacy, University of North Carolina, Chapel Hill, NC 27599, USA; ${ }^{8}$ Chinese Medicine Research and Development Center, China Medical University Hospital, Taichung 404; ${ }^{9}$ Department of Life Sciences, National Chung Hsing University, Taichung 402, Taiwan, R.O.C.; ${ }^{10}$ Department of Biochemistry, Nihon Pharmaceutical University, Saitama 362-0806, Japan
\end{abstract}

Received April 26, 2012; Accepted June 20, 2012

DOI: 10.3892/or.2012.1923

\begin{abstract}
The aim of the present study was to discover the signaling pathways associated with 2-(3-hydroxy-5-methoxyphenyl)-6,7-methylenedioxyquinolin-4-one (YYK1)-induced apoptosis in HL-60 human leukemia cells. YYK1 induced cytotoxic effects, cell morphological changes, decreased the cell number and increased reactive oxygen species (ROS) production and loss of mitochondrial membrane potential $\left(\Delta \Psi_{m}\right)$ in HL-60 cells. YYK1-induced apoptosis was confirmed by the terminal deoxynucleotidyl transferase dUTP nick end labeling (TUNEL) staining. Results from colorimetric assays and western blot analysis indicated that activities of caspase-7/-3, caspase- 8 and caspase-9 were increased in YYK1-treated HL-60 cells. Western blot analysis showed that the protein levels of extrinsic apoptotic proteins (Fas/CD95, FasL and FADD), intrinsic related proteins (cytochrome $c$, Apaf-1, AIF and Endo G), the ratio of Bax/ Bcl-2 and phosphorylated p38 MAPK were increased in HL-60 cells after YYK1 treatment. Cell apoptosis was significantly reduced after pre-treatment with $N$-acetylcysteine (NAC; a ROS scavenger) or diphenyleneiodonium chloride (DPI; a NADPH oxidase inhibitor). Blockage of p38 MAPK signaling
\end{abstract}

Correspondence to: Professor Sheng-Chu Kuo, Graduate Institute of Pharmaceutical Chemistry, China Medical University, No. 91 Hsueh-Shih Road, Taichung 40402, Taiwan, R.O.C.

E-mail: sckuo@mail.cmu.edu.tw

Key words: YYK1, apoptosis, reactive oxygen species, p38 MAPK, HL-60 leukemia cells by SB202190 abolished YYK1-induced Fas/CD95 upregulation and apoptosis in HL-60 cells. We conclude that YYK1 induces both of extrinsic and intrinsic apoptotic pathways via ROS-mediated activation of p38 MAPK signaling in HL-60 human leukemia cells in vitro.

\section{Introduction}

Apoptosis is characterized by morphological changes, cell shrinkage and chromatin condensation (1-3). Two signal pathways are involved in apoptosis. The intrinsic pathway involves disrupting the mitochondrial membrane and then releasing cytochrome $c$, apoptotic protease activating factor-1 (Apaf-1), pro-caspase-9, apoptosis-inducing factor (AIF) and endonuclease $G$ (Endo $G$ ) into the cytosol. The extrinsic pathway through death receptors and ligand interaction such as FasL/Fas and then activate caspase-8 $(4,5)$. Both of intrinsic and extrinsic pathways induced the activation of caspase-7 and caspase-3 (6). A recent study has also demonstrated that the mitogen-activated protein kinases (MAPKs) signaling is able to regulate apoptosis-associated pathways in tumor cells $(7,8)$.

The MAPKs signaling modulate physiological functions and include cell proliferation, differentiation, development and apoptosis $(9,10)$. There are three major subfamilies of MAPKs including extracellular signal-regulated kinase (ERK), c-Jun $N$-terminal kinase (JNK) and p38 proteins (11). Stimulations such as growth factors, hormones, or cytokines activate MAP kinase kinases (MKKs). MKKs phosphorylate tyrosine/ threonine residues of MAPKs then result in dimerization and subsequent activation of MAPKs (12). The ERK cascade is most frequently associated with cell survival activity but the p38 and JNK proteins appear to be pro-apoptotic effects 


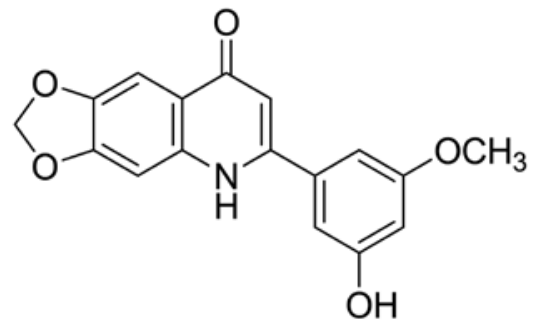

Figure 1. The structure of 2-(3-hydroxy-5-methoxyphenyl)-6,7-methylenedioxyquinolin-4-one (YYK1).

$(11,13)$. p38 MAPK is activated by a variety of cellular stresses and acts at early step prior to dysfunction of mitochondria and caspase activation in cell apoptosis (14). The recent studies have demonstrated that the p38 MAPK kinase cascade is necessary during cell apoptosis by cellular stress $(14,15)$.

In the present study, we designed and synthesized a novel anti-leukemia compound, 2-(3-hydroxy-5-methoxyphenyl)6,7-methylenedioxyquinolin-4-one (YYK1), it is a most potential candidate for anti-leukemia activities as shown in Fig. 1. However, neither the cytotoxic effects of YYK1 on HL-60 leukemia cells nor the molecular mechanisms underlying its anti-leukemia activity have been well investigated. In this study, the proliferation curve showed that YYK1 had time- and concentration-dependent effects on the cytotoxicity of HL-60 cells. YYK1 caused apoptotic cell death, which is preceded by the generation of ROS, sustained activation of $\mathrm{p} 38$ MAPK. Our results strongly suggest a necessary role for the p38 MAPK pathway during cell death by apoptosis in HL-60 cells induced by YYK1.

\section{Materials and methods}

Chemicals and regents. YYK1, 2-(3-hydroxy-5-methoxyphenyl)-6,7-methylenedioxyquinolin-4-one, was synthesized in our lab (Graduate Institute of Pharmaceutical Chemistry, College of Pharmacy, China Medical University). YYK1 was dissolved in sterile DMSO and aliquoted and stored at $-20^{\circ} \mathrm{C}$. Propidium iodide (PI), proteinase K, RNase A, Triton X-100, chemical inhibitors for p38 (SB202109), diphenyleneiodonium chloride (DPI; NADPH oxidase inhibitor) and scavengers of ROS ( $N$-acetylcysteine) were purchased from Sigma-Aldrich Corp. (St. Louis, MO, USA). Sources of antibodies used in this study were: polyclonal antibodies specific for caspase-8, caspase-9, caspase-3 and caspase-7 were obtained from Cell Signaling Technology Inc. (Danvers, MA, USA). Monoclonal antibodies specific for AIF was purchased from Abcam Inc. (Cambridge, MA, USA). Monoclonal antibodies specific for Fas/CD95, FasL, FADD, cytochrome $c$, Apaf-1, Endo G, Bax, $\mathrm{Bcl}-2, \beta$-actin and all peroxidase (HRP)-conjugated secondary antibodies were obtained from Santa Cruz Biotechnology, Inc. (Santa Cruz, CA, USA). Enhanced chemiluminescence (ECL) was purchased from Millipore (Bedford, MA, USA).

Cell culture. The human promyelocytic leukemia cell line (HL-60) was purchased from the Bioresource Collection and Research Centre (BCRC) of the Food Industry Research and Development Institute (Hsinchu, Taiwan). Cells were cultured in RPMI-1640 medium (Gibco/Life Technologies, Carlsbad, CA, USA) and supplemented with $10 \%$ heat-inactivated fetal calf serum (FCS) (HyClone, Logan, UT, USA), 100 Units $/ \mathrm{ml}$ penicillin, $100 \mu \mathrm{g} / \mathrm{ml}$ streptomycin (Gibco/Life Technologies) and $2 \mathrm{mM} \mathrm{L}$-glutamine (Gibco/Life Technologies) at $37^{\circ} \mathrm{C}$ in a $5 \% \mathrm{CO}_{2}$ humidified incubator.

Detection of cell number. HL-60 cells at a density of $2.5 \times 10^{5} /$ well were seeded in 24-well plates and then exposed to 0,125 , 250,500 and $1000 \mathrm{nM}$ of YYK1 or 0.1\% DMSO (as a vehicle control) for 24 and $48 \mathrm{~h}$. Cells were harvested and determined cell number using trypan blue stain by Countess Automated Cell Counter (Invitrogen/Life Technologies) $(16,17)$.

Determinations of cell viability. HL-60 cells $\left(2.5 \times 10^{5} /\right.$ well) in 24-well plates were treated with $125,250,500$ and $1000 \mathrm{nM}$ of YYK1 or $0.1 \%$ DMSO (as a vehicle control) for $48 \mathrm{~h}$. Cells were harvested, washed and re-suspended in phosphate-buffered saline (PBS) buffer containing $4 \mu \mathrm{g} / \mathrm{ml}$ of PI. Cell viability was analyzed using a PI exclusion method and flow cytometry (FACSCalibur, Becton-Dickinson, NJ, USA) equipped with a laser at $488 \mathrm{~nm}$ wavelength $(18,19)$. The percentage of cell viability was calculated as a ratio of the number of YYK1treated cells to that of $0.1 \%$ DMSO as a vehicle-control group. Cell viability was performed in triplicate from three independent experiments.

Cell morphological determination. HL-60 cells at a density $2.5 \times 10^{5} /$ well were seeded in 24-well plates and then exposed to $0,125,250,500$ and $1000 \mathrm{nM}$ of YYK1 or $0.1 \%$ DMSO (as a vehicle control) for $48 \mathrm{~h}$. Cell morphology was examined and photographed under a phase contrast microscope as previously described $(19,20)$.

Analysis for sub-G1 phase distribution. HL-60 cells (2.5x10 $/$ well) were placed in 24-well plates and incubated with 125 , 250,500 and $1000 \mathrm{nM}$ of YYK1 or vehicle control $(0.1 \%$ DMSO) for $48 \mathrm{~h}$. For determination of cell apoptosis at sub-G1 phase, cells were fixed gently by putting $70 \%$ ethanol at $-20^{\circ} \mathrm{C}$ overnight, and then re-suspended in PBS containing $40 \mu \mathrm{g} / \mathrm{ml}$ of PI, $0.1 \mathrm{mg} / \mathrm{ml}$ R Nase A and $0.1 \%$ Triton X-100 in dark room for $30 \mathrm{~min}$. Cell cycle distribution and apoptotic nuclei were determined by flow cytometry as previously described $(21,22)$.

TUNEL assay. HL-60 cells $\left(2.5 \times 10^{5}\right.$ cells/well) in $24-w e l l$ plates were exposed to $125,250,500$ and $1000 \mathrm{nM}$ of YYK1 or $0.1 \%$ DMSO (as a vehicle control) for $48 \mathrm{~h}$. For the specific inhibitor assay, cells were pretreated with $2 \mathrm{mM}$ of NAC, $10 \mu \mathrm{M}$ of DPI and $10 \mu \mathrm{M}$ of SB202190, respectively for $1 \mathrm{~h}$, followed by treatment with or without $500 \mathrm{nM}$ of YYK1. After incubation for $48 \mathrm{~h}$, in situ apoptosis detection of DNA fragmentation was determined by using the terminal deoxyribonucleotide transferase-mediated dUTP nick endlabeling (TUNEL) assay kit (In Situ Cell Death Detection kit, Fluorescein, Roche Diagnostics, Hillsdale, MI, USA). Following TUNEL staining, samples were washed once and re-suspended in $0.5 \mathrm{ml}$ of PBS containing $10 \mu \mathrm{g} / \mathrm{ml}$ of PI. TUNEL positive cells were analyzed by flow cytometry as previously described $(1,23)$. The median fluorescence intensity 
was quantified by BD CellQuest Pro software. TUNEL assays were performed in triplicate in three independent experiments.

Assays for caspase activities. HL-60 cells at a density of $1 \times 10^{7} /$ dish were seeded in 10-cm dishes and then exposed to 125 , 250, 500 and $1000 \mathrm{nM}$ of YYK1 or 0.1\% DMSO (as a vehicle control) for $48 \mathrm{~h}$. Cells were lysed in lysis buffer [50 mM Tris-HCl (pH 7.4), 1 mM EDTA, 10 mM EGTA, 10 mM digitonin and $2 \mathrm{mM}$ DTT]. About $50 \mu \mathrm{g}$ of cytosol proteins were incubated with caspase- 9 , caspase-8, caspase-7/-3 specific substrates (R\&D Systems) for $1 \mathrm{~h}$ at $37^{\circ} \mathrm{C}$. The caspase activity was determined by measuring OD405 as previously described $(18,24)$.

Determination of reactive oxygen species (ROS) and mitochondrial membrane potential $(\Delta \Psi \mathrm{m})$. HL-60 cells at a density of $2.5 \times 10^{5} /$ well were plated in 24 -well plates and then exposed to $125,250,500$ and $1000 \mathrm{nM}$ of YYK1 or $0.1 \%$ DMSO (as a vehicle control) for $6 \mathrm{~h}$. Cells were harvested then washed twice by PBS, and then re-suspended in 2,7-Dichlorodihydrofluorescein diacetate (DCFH-DA; $10 \mu \mathrm{M})$ for ROS determination and in $\mathrm{DiOC}_{6}(500 \mathrm{nM})$ for $\Delta \Psi \mathrm{m}$. Cells were incubated for $30 \mathrm{~min}$ at $37^{\circ} \mathrm{C}$ in the dark room and analyzed immediately by flow cytometry as previously described $(19,25)$.

Western blot analysis. HL-60 cells ( $1 \times 10^{7}$ cells/dish) in $10-\mathrm{cm}$ dishes were treated with or without $125,250,500$ and $1000 \mathrm{nM}$ of YYK1 or $0.1 \%$ DMSO (as a vehicle control) for $48 \mathrm{~h}$. Total protein and cytosolic fraction were prepared and lysated in the into the PRO-PREP ${ }^{\mathrm{TM}}$ Protein Extraction solution (iNtRON Biotechnology, Gyeonggi-do, Korea). Equal amounts of $30 \mu \mathrm{g}$ protein were separated by $10-12 \%$ sodium dodecyl sulfate polyacrylamide gel electrophoresis (SDS-PAGE) and electrotransferred to a nitrocellulose membrane by using BBot $^{\mathrm{TM}}$ Dry Blotting System (Invitrogen/Life Technoligies). Blots were blocked in PBST buffer $(0.05 \%$ Triton X-100 in PBS) containing 5\% non-fat milk for $1 \mathrm{~h}$, and then incubated with specific primary antibodies at $4^{\circ} \mathrm{C}$ overnight. The membrane was washed with PBST buffer and incubated with secondary antibodies conjugated horseradish peroxidase (HRP). The specific protein was detected by using Immobilon Western Chemiluminescent HRP substrate (Millipore, Bedford, MA, USA) as previously described $(18,26,27)$.

Statistical analysis. Data are presented as the mean \pm SEM for the indicated number of separate experiment. Statistical analyses of data were done by Student's t-test, and ${ }^{*} \mathrm{P}<0.05$, ${ }^{* * *} \mathrm{P}<0.001$ were considered significant.

\section{Results}

YYK1 inhibits cell number and viability in HL-60 cells. To assess the effect of YYK1 on cell number, HL-60 cells were exposed to $0,125,250,500$ and $1000 \mathrm{nM}$ of YYK1 for 24 and $48 \mathrm{~h}$ and determined cell number using trypan blue stain by Countess Automated Cell Counter (Invitrogen/Life Technologies). As shown in Fig. 2A, YYK1 inhibited cell number of HL-60 cells in a concentration- and time-dependent manner. To investigate the effect of YYK1 on cell viability,
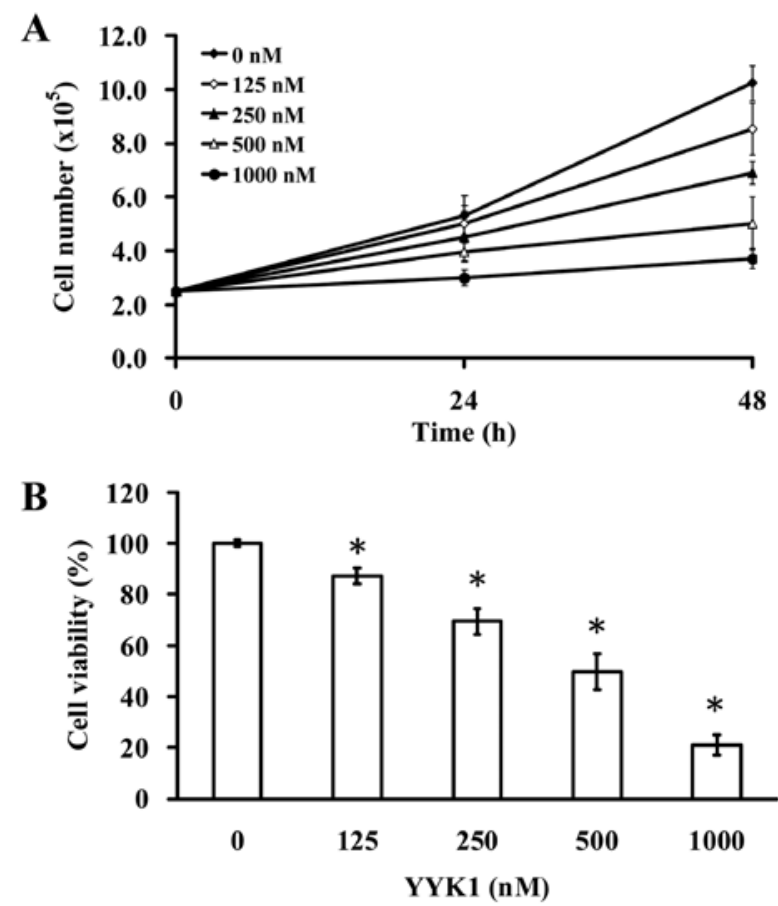

Figure 2. Effects of YYK1 on cell number and viability in the human leukemia cell line (HL-60). HL-60 cells were treated with 0, 125, 250, 500 and $1000 \mathrm{nM}$ of YYK1 for indicated intervals of time. (A) After 24 and $48 \mathrm{~h}$ exposures, cell number was investigated utilizing Countess Automated Cell Counter (Invitrogen/Life Technologies). (B) After 48-h treatment, cell viability was determined by a PI exclusion method and flow cytometry. The data are shown as the mean \pm SEM of three independent experiments. ${ }^{*} \mathrm{P}<0.05$ vs untreated control.

HL-60 cells were treated with $125,250,500$ and $1000 \mathrm{nM}$ of YYK1 for $48 \mathrm{~h}$ and measured cell viability using a PI exclusion assay and flow cytometric analysis. Fig. 2B shows that YYK1 concentration-dependently reduced cell viability of HL-60 cells. The half maximal inhibitory concentration $\left(\mathrm{IC}_{50}\right)$ for $48 \mathrm{~h}$ of treatment of YYK1 in HL-60 cells was $521.39 \pm 3.25 \mathrm{nM}$.

YYK1 induces cell apoptosis in HL-60 cells. To verify YYK1induced cytotoxicity, we examined the cell morphological changes in HL-60 cell after exposure to 125, 250, 500 and $1000 \mathrm{nM}$ of YYK1 for $48 \mathrm{~h}$. Results in Fig. 3A display that YYK-1 induced characteristic features of cell shrinking and rounding as well as formation of apoptotic bodies. Further studies investigated the possible mechanisms focusing on cell cycle arrest or cell death by inhibitory effects in YYK1treated HL-60 cells. Our results demonstrated that YYK1 induced sub-G1 phase (cell death) at 48 -h treatments and these effects occurred in a concentration-dependent manner. To verify the cell apoptosis in YYK1-treated HL-60 cells, we assessed the DNA fragmentation by TUNEL staining. As shown in Fig. 3C, YYK1 induced DNA fragmentation (TUNEL positive cells) in HL-60 cells, which was increased in a concentration-dependent manner. Our results suggest that YYK-1 induced DNA fragmentation for cell apoptosis in HL-60 cells.

YYK1 stimulates the activities of caspase-3/-7, caspase- 8 and caspase-9 in HL-60 cells. To examine whether caspases 
A

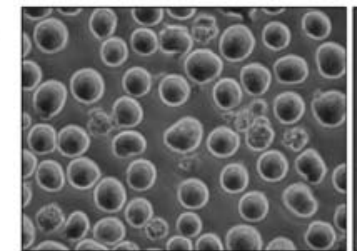

Control

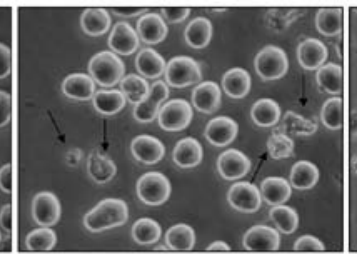

YYK1 (250 nM)

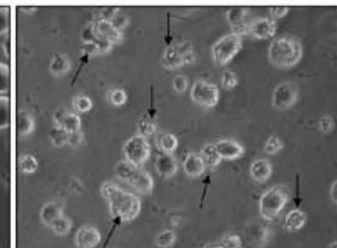

YYK1 (500 nM)

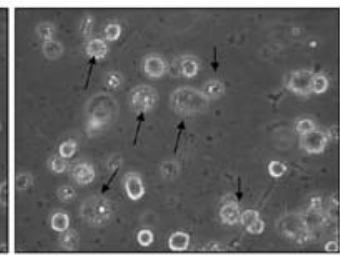

YYK1 (1000 nM)
B

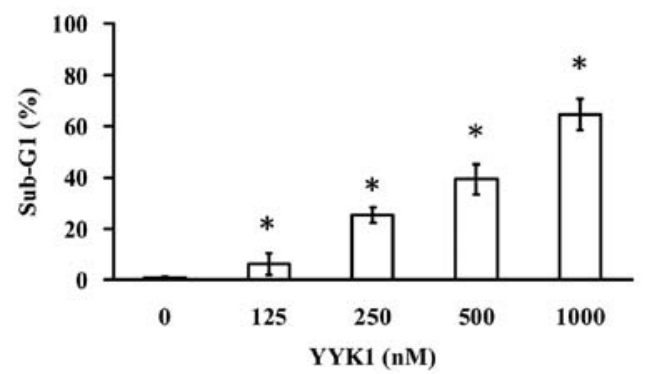

C

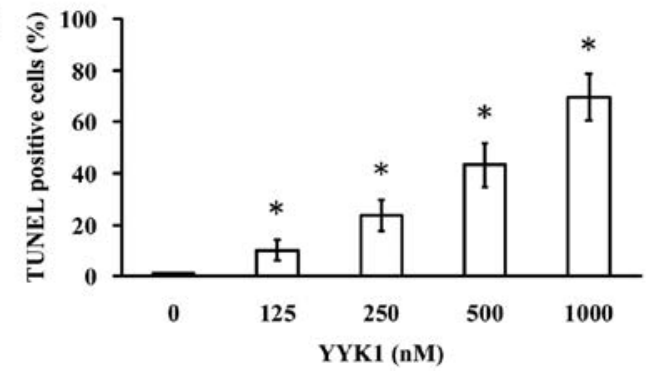

Figure 3. YYK1 affects morphological changes and induced apoptosis in HL-60 cells. (A) Cells were exposed to various concentrations (0, 250, 500 and $1000 \mathrm{nM}$ ) of YYK1 for $48 \mathrm{~h}$ and then photographed under a phase-contrast microscope at x200 magnification. For detecting the apoptotic death, cells were treated with $0,125,250,500$ and 1000 of YYK1 for 48 h, and stained by PI and TUNEL assay (In Situ Cell Death Detection Kit, Fluorescein, Roche Diagnostics), respectively, by flow cytometry as described in Materials and methods to determine (B) sub-G1 population (apoptosis) and (C) DNA fragmentation. The data shown are the mean \pm SEM of three independent experiments. ${ }^{*} \mathrm{P}<0.05$ vs. untreated control.

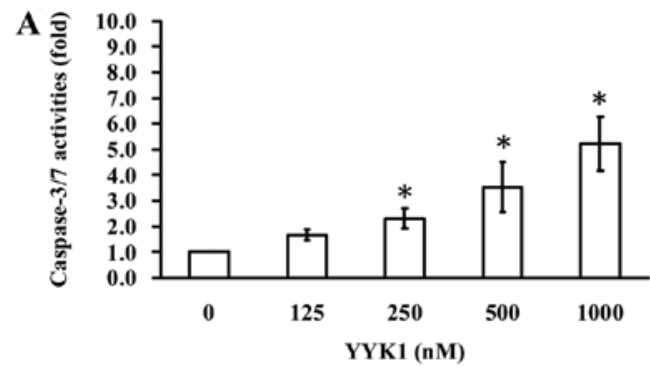

C
B

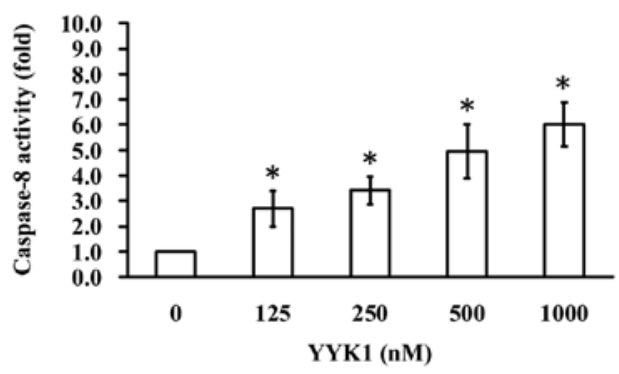

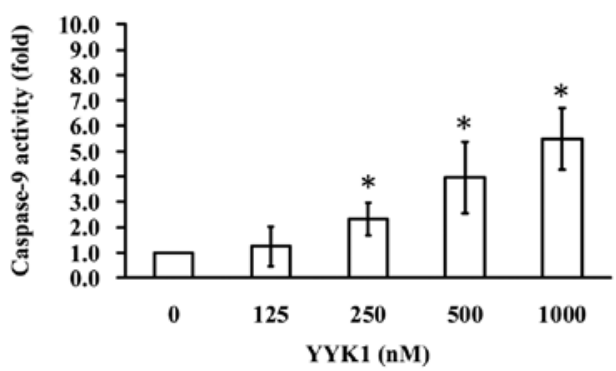

Figure 4. YYK1 promotes the caspase-3/7, caspase-8 and caspase-9 activities in HL-60 cells. Cells were treated with 0, 125, 250,500 and $1000 \mathrm{nM}$ of YYK1 for $48 \mathrm{~h}$ and the whole-cell lysate was subjected to (A) caspase-3/7, (B) caspase- 8 and (C) caspase- 9 activities as described in Materials and methods. The data shown are the mean \pm SEM of three independent experiments. ${ }^{*} \mathrm{P}<0.05$ vs. the vehicle-control sample.

activities are involved in YYK1-induced apoptosis in HL-60 cells, cells were exposed to $125,250,500$ and $1000 \mathrm{nM}$ of YYK1 for $48 \mathrm{~h}$ and then the activities of caspase-3/-7, -8 and -9 were determined by colorimetric assays. Results demonstrated that YYK1 promoted the activation of caspase-3/-7 (Fig. 4A), caspase-8 (Fig. 4B) and caspase-9 (Fig. 4C) after 48 h treatment. Based on these observations, the YYK1-induced cell death may be mediated through extrinsic and intrinsic apoptotic pathways in HL-60 cells.
YYK1 induces reactive oxygen species ( $R O S)$ production and loss of $\Delta \Psi \mathrm{m}$ in $H L-60$ cells. We further investigated the upstream signals of YYK1-induced apoptosis in HL-60 cells. Cells were exposed to $125,250,500$ and $1000 \mathrm{nM}$ of YYK1 for $6 \mathrm{~h}$, and then measurement in the level of ROS production by flow cytometric assay. YYK1-promoted the ROS production is a concentration-dependent effect as seen in Fig. 5A. We also examined the effects of YYK1 on the $\Delta \Psi \mathrm{m}$. Our data indicated that YYK1 decreased $\Delta \Psi_{m}$ (Fig. 5B) after $6 \mathrm{~h}$ 
A

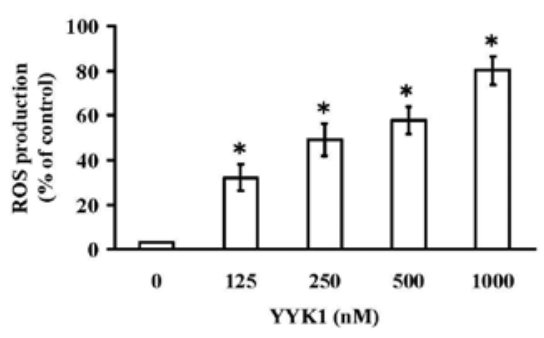

B

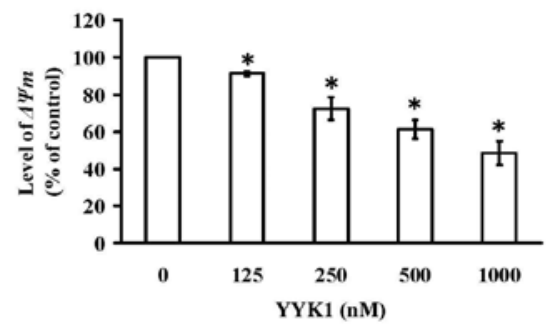

C

Figure 5. YYK1 enhanced the ROS production and loss of $\Delta \Psi m$ in HL-60 cells. Cells were treated with $0,125,250,500$ and $1000 \mathrm{nM}$ of YYK1 for $6 \mathrm{~h}$. The levels of (A) ROS production and (B) $\triangle \Psi$ m were stained with 2,7-dichlorodihydrofluorescein diacetate (DCFH-DA) and DiOC 6 , respectively. The stained cells were determined by flow cytometry as described in Materials and methods. (C) Cells were pretreated with or without $2 \mathrm{mM}$ of NAC, $10 \mu \mathrm{M}$ of DPI, respectively for $1 \mathrm{~h}$ and then were treated with $500 \mathrm{nM}$ of YYK1 for $48 \mathrm{~h}$. The apoptotic cells were determined by TUNEL assay and analyzed by flow cytometry as described in Materials and methods. The data shown are the mean \pm SEM of three independent experiments. ${ }^{*} \mathrm{P}<0.05$ vs. untreated control. ${ }^{\#} \mathrm{P}<0.05$ vs. $\mathrm{Y} Y \mathrm{~K} 1$ treatment for $48 \mathrm{~h}$.

A

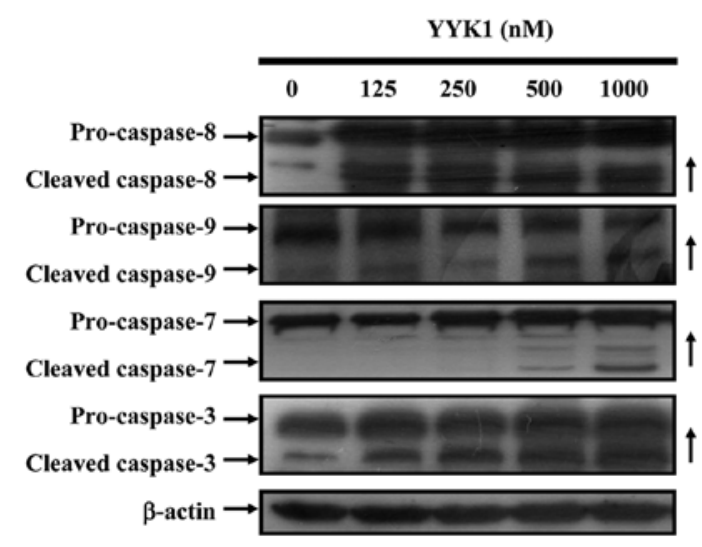

B

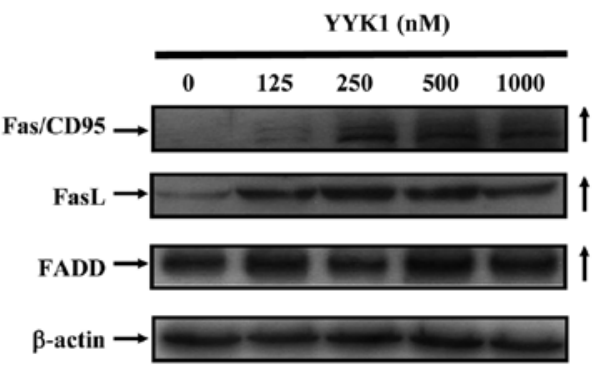

C

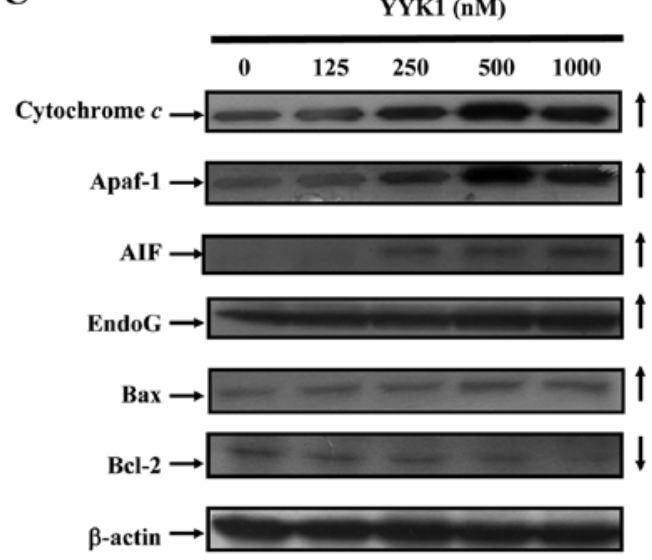

Figure 6. YYK1 altered the apoptosis-associated protein levels of intrinsic and extrinsic signaling in HL-60 cells. Cells were exposed to 0, 125, 250, 500 and $1000 \mathrm{nM}$ of YYK1 for $48 \mathrm{~h}$, and then harvested total protein lysed for the detection the protein levels by western blot analysis. (A) The protein levels of caspase-8, caspase-9, caspase-7 and caspase-3; (B) Fas/CD95, FasL and FADD; (C) cytochrome $c$, Apaf-1, AIF, Endo G, Bax and Bcl-2. $\beta$-actin is the internal control. The data present are from three individual experiments with similar results.

treatment in HL-60 cells. Importantly, we found decreased percentage of apoptotic cells (TUNEL positive cells) when compared with untreated cells, when cells were pretreated with $\mathrm{N}$-acetylcysteine (NAC, ROS scavenger) or diphenylenei- 
A

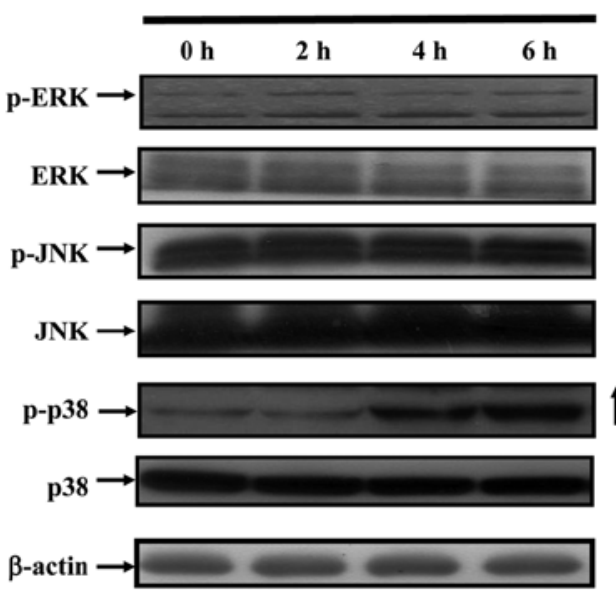

B

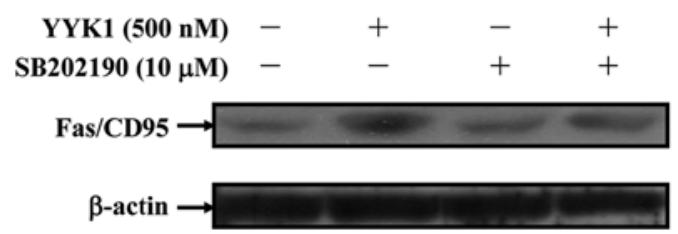

C

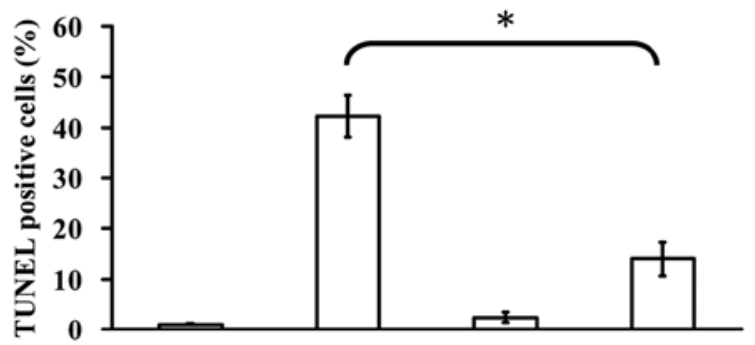

YYK1 (500 nM) $\quad-\quad+\quad+\quad+$ SB202190 (10 $\mu \mathrm{M}) \quad-\quad \quad \quad-\quad \quad+\quad+$

Figure 7. YYK1 affects MAPK signaling-associated protein levels and Fas/CD95 signaling in HL-60 cells. Cells were pre-incubated with or without $10 \mu \mathrm{M}$ of SB202190 (a selective inhibitor of p38 MAPK) and then exposed to $500 \mathrm{nM}$ of YYK1 for 0, 2, 4 and $6 \mathrm{~h}$. At the end of treatment, cell lysate was collected to subject to the protein level by western blot analysis. (A) The protein levels of p-ERK, ERK, p-JNK, JNK, p-p38 and p38. The anti- $\beta$-actin was used as a loading control. Similar results were obtained in three independent experiments. (B) The protein levels of Fas/CD95 were examined by western blot analysis and (C) TUNEL assay after pre-treating with SB202190 in YKK1-treated HL-60 cells for $48 \mathrm{~h}$. The data shown are the mean \pm SEM of three independent experiments. ${ }^{*} \mathrm{P}<0.05$ vs. YYK1 treatment for $48 \mathrm{~h}$.

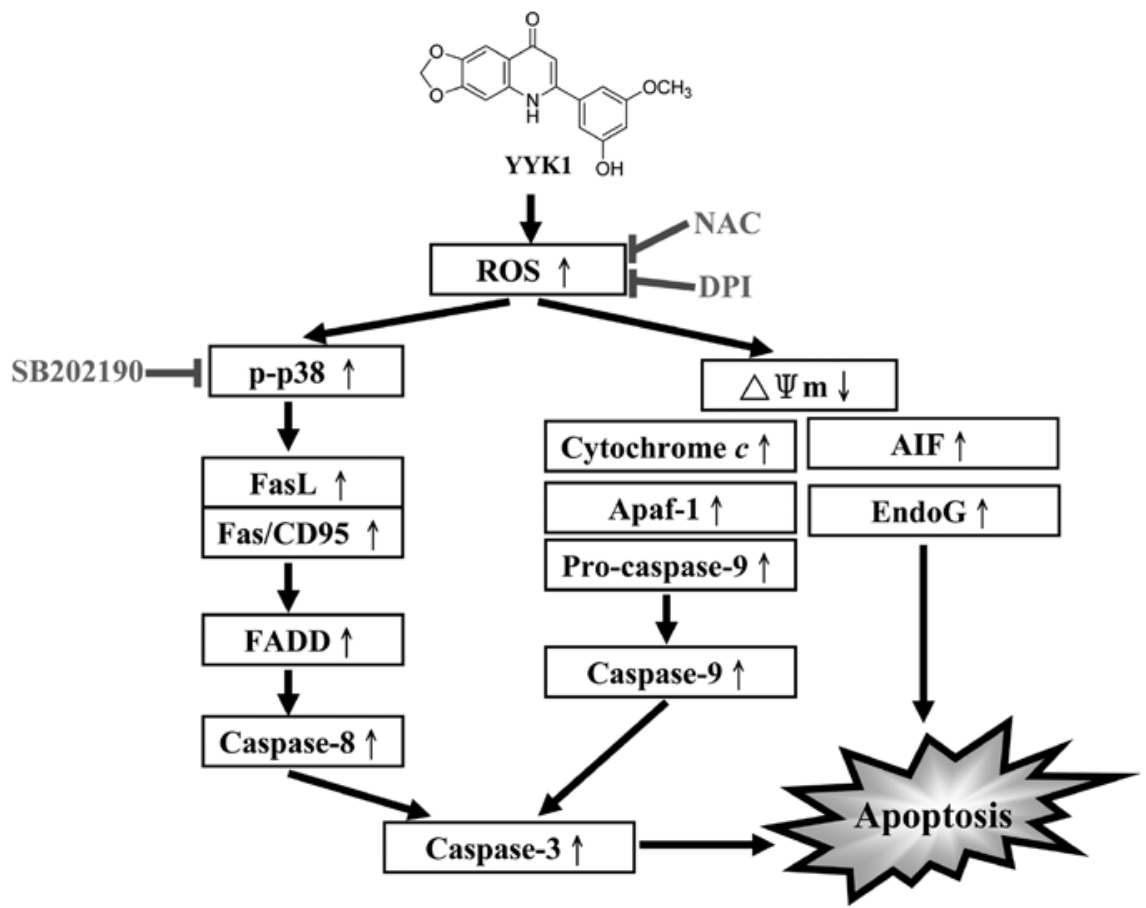

Figure 8. The proposed signaling network shows the YYK1 provokes cell apoptosis through stimulating ROS generations and upregulation of p38 signaling pathway in HL-60 human leukemia cells.

odonium chloride (DPI; NADPH oxidase inhibitor) and then treated with YYK1 (Fig. 5C). Thus, we suggest that YYK1induced apoptosis is involved in ROS production and loss of $\Delta \Psi m$ in HL-60 cells.
YYK1 affects apoptosis-associated protein levels (intrinsic and extrinsic signaling) in $\mathrm{HL}-60$ cells. We investigated the apoptosis-associated protein levels for apoptotic death by western blotting. As illustrated in Fig. 6A, YYK1 caused an 
increase in the protein level of cleavage-caspase- 8 , cleavagecaspase- 9 , cleavage-caspase- 7 and cleavage-caspase- 3 in HL-60 cells. Results shown in Fig. 6B indicate that YYK1 increased the death receptor pathway-associated protein levels, including Fas/CD95, FasL, FADD. Furthermore, mitochondrial pathway-related protein levels (cytochrome $c$, Apaf-1, ALF, Endo G and Bax) were increased but the level of Bcl-2 was decreased in YYK1-treated HL-60 cells (Fig. 6C). Our results indicated that YYK1-induced apoptosis may affect intrinsic and extrinsic signaling-associated proteins in HL-60 cells.

YYK1 stimulates the activity of $p 38$ MAPK and upregulated the expression of Fas/CD95 in HL-60 cells. To examine the involvement of MAPKs pathway in YYK1-regulated apoptosis in HL-60 cells, we investigated the phosphorylated MAPK (phospho-ERK, phospho-JNK and phospho-p38) protein levels by western blot analysis. YYK1 promoted an increase in protein levels of phospho-p38 in HL-60 cells as shown in Fig. 7A. It is reported that Fas/CD95 is a major regulator of p38 MAPK signaling $(28,29)$, we next determined the protein levels of Fas/CD95 by western blot analysis in YYK1-treated HL-60 cells after pretreatment with p38 MAPK inhibitor (SB202190). When cells were pretreated with SB20219 and then treated with YYK1, our results showed that the protein level of Fas/CD95 was decreased (Fig. 7B), and the percentage of apoptotic cells abolished (TUNEL positive cells) when compared untreated cells (Fig. 7C). Therefore, we found that p38 MAPK contributes to the YYK1-induced Fas/CD95 protein expression and apoptotic death in HL-60 cell apoptosis.

\section{Discussion}

In our early studies, we demonstrated that the 6,7-methylenedioxyquinolin-4-one derivative CHM-1 is a potential compound for anticancer activities (30-35). CHM-1 significantly inhibited tubulin polymerization and showed cytotoxic effect on many human tumor cell lines (SK-Hep-1, HA22T, Hep3B, HepG2, U2-OS cells and CT26 cells) in vitro (30,31,33-36) and its phosphate (CHM-1-P) inhibited CT26 tumor growth in vivo $(32,33)$. Induction of apoptosis through p53/DR5 upregulation by CHM-1 has been demonstrated in human umbilical vein endothelial cells (HUVECs) (37). In recent years, we have designed and synthesized a new series of 6,7-methylenedioxyquinolin-4-one derivatives as new anti-leukemia agents and YYK1 (Fig. 1) is the most potential compound against HL-60 leukemia cells. However, the cytotoxic effects of YYK1 on HL-60 leukemia cells and the anti-leukemia mechanisms are not fully clarified. We first demonstrated that YYK1 induced cytotoxic effects through induction of apoptosis in HL-60 cells. YYK1 could be used as a novel therapeutic agent for the treatment of leukemia in the future.

Our results showed the increased sub-G1 population by DNA content analysis may involve total cell death, including apoptosis and necrosis (Fig. 3B). To determine if the sub-G1 population was due to apoptotic cell death after exposure to YYK1, a quantitative assessment of apoptosis was detected by TUNEL staining analysis. YYK1 treatment caused an increase in TUNEL positive cells (Fig. 3C), indicating the cytotoxic activity of YYK1 was apoptotic and not necrotic death. YYK1 induced the activation of caspase-9, caspase-8, caspase-7/-3 after 48-h treatment (Fig. 4), and western blot analysis was also used to confirm the significant increases in the protein levels of cleaved caspase-3, cleaved caspase-8, and cleaved caspase-9 after YYK1 treatment (Fig. 6A). Our results suggested that YYK1 may induce intrinsic and extrinsic apoptotic pathways. Our previous reports demonstrate that the 6,7-methylenedioxyquinolin-4-one series compound CHM-1, induced apoptosis via a ROS-dependent mitochondrial death pathway in human osteogenic sarcoma U-2 OS cells (36). YYK1 promoted the ROS production in HL-60 cells (Fig. 5A). Moreover, HL-60 cells were pretreated with $N$-acetylcysteine (NAC, a ROS scavenger) or diphenyleneiodonium chloride (DPI; NADPH oxidase inhibitor), respectively, which led to decrease in the apoptotic cells in comparison to the only YYK1-treated cells (Fig. 5C). Our results proposed that ROS production may be involved in YYK1-induced apoptotic cell death in HL-60 cells. We demonstrated that an increase of ROS production after YYK1 treatment for $6 \mathrm{~h}$ (Fig. 7), and YYK1 then promoted the Fas/CD95, FasL, FADD (Fig. 6B) and releases of cytochrome $c$, Apaf-1 and AIF protein levels from mitochondria into the cytosol (Fig. 6C) in YYK1-treated HL-60 cells. Based on the evidence in Fig. 6, we suggest that YYK1-stimulated cell death is involved in the intrinsic and extrinsic apoptotic pathway.

Following YYK1 treatment, the production of phosphorylated p38 (p-p38) was shown in YKK1-treated HL-60 cells but the protein levels of p-ERK and p-JNK were no significant compared with the control cells. In contrast, YYK1 induced the protein levels of p-p38 at 2, 4 and $6 \mathrm{~h}$ (Fig. 7A), suggesting that p38 MAPK activation is critical during YYK1-induced cell apoptosis. p38 MAPK is a stress-activated MAP kinase that is preferentially activated by cell stress-inducing signals, including oxidative stress and cytotoxic chemical agent (38-40). In the present study, YYK1-induced phosphorylation of p38 MAPK was abrogated by pretreatment with NAC, suggesting that ROS were upstream events for p38 MAPK activation (data not shown). Previous studies suggest that p38 MAPK is involved in upregulating Fas/ CD95 expression $(41,42)$. Our study focused on confirming this hypothesis using SB202190 (p38 MAPK inhibitor) in YKK1-treated HL-60 cells. As shown in Fig. 7B, SB202190 abrogated YYK1-induced expression of Fas/CD95 in HL-60 cells. Moreover, TUNEL analysis revealed that $43.96 \%$ of the HL-60 cells were apoptotic cells after 48-h treatment with YYK1. However, only $14.29 \%$ was apoptotic cells in the YYK1 treatment (Fig. 7C) when the HL-60 cells were pre-treated with SB202190 $(10 \mu \mathrm{M})$ of for $1 \mathrm{~h}$. In this study, we demonstrated an important role for p38 MAPK and its link between ROS generation and Fas/CD95-mediated apoptosis in YYK1-treated HL-60 cells. Taken together, the involvement of p38 MAPK activation in upregulation of Fas/CD95 protein expression and activations of intrinsic and extrinsic apoptotic pathways are proposed in YYK1treated cells (Fig. 8). In this regard, understanding the signal pathways responsible for YYK1-induced apoptosis might lead to discovering more effective strategies in improving leukemia treatment. 


\section{Acknowledgements}

The study was supported by research grants from the National Science Council of the Republic of China awarded to S.-C.K. (NSC 100-2320-B-039-001) and L.-J.H. (NSC 95-2320-B-039011-MY3). Experimental and data analysis were performed in part through the use of the Medical Research Core Facilities Center, Office of Research and Development, China Medical University, Taichung, Taiwan, R.O.C. This study also was supported by the Taiwan Department of Health, China Medical University Hospital Cancer Research Center of Excellence (DOH101-TD-C-111-005).

\section{References}

1. Chung JG, Yang JS, Huang LJ, et al: Proteomic approach to studying the cytotoxicity of YC-1 on U937 leukemia cells and antileukemia activity in orthotopic model of leukemia mice. Proteomics 7: 3305-3317, 2007.

2. Sanjiv K, Su TL, Suman S, et al: The novel DNA alkylating agent BO-1090 suppresses the growth of human oral cavity cancer in xenografted and orthotopic mouse models. Int J Cancer 130 1440-1450, 2012.

3. Huang SM, Yang JS, Tsai SC, et al: The novel synthesized 2-(3-(methylamino)phenyl)-6-(pyrrolidin-1-yl)quinolin-4-one (Smh-3) compound induces G2/M phase arrest and mitochondrial-dependent apoptotic cell death through inhibition of CDK1 and AKT activity in HL-60 human leukemia cells. Int J Oncol 38: 1357-1364, 2011.

4. Lavrik IN, Golks A and Krammer PH: Caspases: pharmacological manipulation of cell death. J Clin Invest 115: 2665-2672, 2005.

5. Orrenius S: Reactive oxygen species in mitochondria-mediated cell death. Drug Metab Rev 39: 443-455, 2007.

6. Lombard C, Nagarkatti M and Nagarkatti PS: Targeting cannabinoid receptors to treat leukemia: role of cross-talk between extrinsic and intrinsic pathways in Delta9-tetrahydrocannabinol (THC)-induced apoptosis of Jurkat cells. Leuk Res 29: 915-922, 2005.

7. Ortiz MA, Lopez-Hernandez FJ, Bayon Y, Pfahl M and Piedrafita FJ: Retinoid-related molecules induce cytochrome c release and apoptosis through activation of c-Jun $\mathrm{NH}(2)$-terminal kinase/p38 mitogen-activated protein kinases. Cancer Res 61: 8504-8512, 2001.

8. Chuang SM, Wang IC and Yang JL: Roles of JNK, p38 and ERK mitogen-activated protein kinases in the growth inhibition and apoptosis induced by cadmium. Carcinogenesis 21: 1423-1432, 2000.

9. Kim EK and Choi EJ: Pathological roles of MAPK signaling pathways in human diseases. Biochim Biophys Acta 1802: 396-405, 2010.

10. Wagner EF and Nebreda AR: Signal integration by JNK and p38 MAPK pathways in cancer development. Nat Rev Cancer 9: 537-549, 2009.

11. Roux PP and Blenis J: ERK and p38 MAPK-activated protein kinases: a family of protein kinases with diverse biological functions. Microbiol Mol Biol Rev 68: 320-344, 2004.

12. Kyriakis JM and Avruch J: Mammalian mitogen-activated protein kinase signal transduction pathways activated by stress and inflammation. Physiol Rev 81: 807-869, 2001.

13. Liu J and Lin A: Role of JNK activation in apoptosis: a doubleedged sword. Cell Res 15: 36-42, 2005.

14. Choi SY, Kim MJ, Kang CM, et al: Activation of Bak and Bax through c-abl-protein kinase Cdelta-p38 MAPK signaling in response to ionizing radiation in human non-small cell lung cancer cells. J Biol Chem 281: 7049-7059, 2006.

15. Zhang W and Liu HT: MAPK signal pathways in the regulation of cell proliferation in mammalian cells. Cell Res 12: 9-18, 2002.

16. Duan X, Ponomareva L, Veeranki S and Choubey D: IFI16 induction by glucose restriction in human fibroblasts contributes to autophagy through activation of the ATM/AMPK/p53 pathway. PLoS One 6: e19532, 2011.
17. Chen HM, Chang FR, Hsieh YC, et al: A novel synthetic protoapigenone analogue, WYC02-9, induces DNA damage and apoptosis in DU145 prostate cancer cells through generation of reactive oxygen species. Free Radic Biol Med 50: 1151-1162, 2011.

18. Yang JS, Hour MJ, Huang WW, Lin KL, Kuo SC and Chung JG: MJ-29 inhibits tubulin polymerization, induces mitotic arrest, and triggers apoptosis via cyclin-dependent kinase 1-mediated Bcl-2 phosphorylation in human leukemia U937 cells. J Pharmacol Exp Ther 334: 477-488, 2010.

19. Lu CC, Yang JS, Huang AC, et al: Chrysophanol induces necrosis through the production of ROS and alteration of ATP levels in J5 human liver cancer cells. Mol Nutr Food Res 54: 967-976, 2010.

20. Kuo TC, Yang JS, Lin MW, et al: Emodin has cytotoxic and protective effects in rat C6 glioma cells: roles of Mdrla and nuclear factor kappaB in cell survival. J Pharmacol Exp Ther 330: 736-744, 2009.

21. Wu SH, Hang LW, Yang JS, et al: Curcumin induces apoptosis in human non-small cell lung cancer NCI-H460 cells through ER stress and caspase cascade- and mitochondria-dependent pathways. Anticancer Res 30: 2125-2133, 2010.

22. Ji BC, Hsu WH, Yang JS, et al: Gallic acid induces apoptosis via caspase- 3 and mitochondrion-dependent pathways in vitro and suppresses lung xenograft tumor growth in vivo. J Agric Food Chem 57: 7596-7604, 2009.

23. Wu PP, Liu KC, Huang WW, et al: Triptolide induces apoptosis in human adrenal cancer NCI-H295 cells through a mitochondrialdependent pathway. Oncol Rep 25: 551-557, 2011.

24. Huang WW, Chiu YJ, Fan MJ, et al: Kaempferol induced apoptosis via endoplasmic reticulum stress and mitochondriadependent pathway in human osteosarcoma U-2 OS cells. Mol Nutr Food Res 54: 1585-1595, 2010.

25. Chiang JH, Yang JS, Ma CY, et al: Danthron, an anthraquinone derivative, induces DNA damage and caspase cascades-mediated apoptosis in SNU-1 human gastric cancer cells through mitochondrial permeability transition pores and Bax-triggered pathways. Chem Res Toxicol 24: 20-29, 2011.

26. Huang WW, Ko SW, Tsai HY, et al: Cantharidin induces G2/M phase arrest and apoptosis in human colorectal cancer colo 205 cells through inhibition of CDK1 activity and caspase-dependent signaling pathways. Int J Oncol 38: 1067-1073, 2011.

27. Chiu YJ, Hour MJ, Lu CC, et al: Novel quinazoline HMJ-30 induces U-2 OS human osteogenic sarcoma cell apoptosis through induction of oxidative stress and up-regulation of ATM/ p53 signaling pathway. J Orthop Res 29: 1448-1456, 2011.

28. Cardier JE and Erickson-Miller CL: Fas (CD95)- and tumor necrosis factor-mediated apoptosis in liver endothelial cells: role of caspase-3 and the p38 MAPK. Microvasc Res 63: 10-18, 2002.

29. Ivanov VN and Ronai Z: p38 protects human melanoma cells from UV-induced apoptosis through down-regulation of NF-kappaB activity and Fas expression. Oncogene 19: 3003-3012, 2000.

30. Lee JC, Chou LC, Huang CH, et al: CHM-1 induces apoptosis via p38-mediated upregulation of DR5 expression in human ovarian cancer SKOV3 cells. Eur J Pharmacol 670: 96-104, 2011.

31. Chen HY, Lu HF, Yang JS, et al: The novel quinolone CHM-1 induces DNA damage and inhibits DNA repair gene expressions in a human osterogenic sarcoma cell line. Anticancer Res 30: 4187-4192, 2010

32. Chou LC, Chen CT, Lee JC, et al: Synthesis and preclinical evaluations of 2-(2-fluorophenyl)-6,7-methylenedioxyquinolin-4-one monosodium phosphate (CHM-1-P-Na) as a potent antitumor agent. J Med Chem 53: 1616-1626, 2010.

33. Chou LC, Yang JS, Huang LJ, et al: The synthesized 2-(2-fluorophenyl)-6,7-methylenedioxyquinolin-4-one (CHM-1) promoted $\mathrm{G} 2 / \mathrm{M}$ arrest through inhibition of CDK1 and induced apoptosis through the mitochondrial-dependent pathway in CT-26 murine colorectal adenocarcinoma cells. J Gastroenterol 44: 1055-1063, 2009.

34. Wang SW, Pan SL, Huang YC, et al: CHM-1, a novel synthetic quinolone with potent and selective antimitotic antitumor activity against human hepatocellular carcinoma in vitro and in vivo. Mol Cancer Ther 7: 350-360, 2008.

35. Wang SW, Pan SL, Peng CY, et al: CHM-1 inhibits hepatocyte growth factor-induced invasion of SK-Hep-1 human hepatocellular carcinoma cells by suppressing matrix metalloproteinase- 9 expression. Cancer Lett 257: 87-96, 2007. 
36. Hsu SC, Yang JS, Kuo CL, et al: Novel quinolone CHM-1 induces apoptosis and inhibits metastasis in a human osterogenic sarcoma cell line. J Orthop Res 27: 1637-1644, 2009.

37. Tsai AC, Pan SL, Sun HL, et al: CHM-1, a new vascular targeting agent, induces apoptosis of human umbilical vein endothelial cells via p53-mediated death receptor 5 up-regulation. J Biol Chem 285: 5497-5506, 2010.

38. Benhar M, Engelberg D and Levitzki A: ROS, stress-activated kinases and stress signaling in cancer. EMBO Rep 3: 420-425, 2002.

39. Mates JM, Segura JA, Alonso FJ and Marquez J: Intracellular redox status and oxidative stress: implications for cell proliferation, apoptosis, and carcinogenesis. Arch Toxicol 82: 273-299, 2008.
40. Kim GS, Choi YK, Song SS, Kim WK and Han BH: MKP-1 contributes to oxidative stress-induced apoptosis via inactivation of ERK1/2 in SH-SY5Y cells. Biochem Biophys Res Commun 338: 1732-1738, 2005.

41. Singhal PC, Bhaskaran M, Patel J, et al: Role of p38 mitogenactivated protein kinase phosphorylation and Fas-Fas ligand interaction in morphine-induced macrophage apoptosis. J Immunol 168: 4025-4033, 2002.

42. Morley SJ, McKendrick L and Bushell M: Cleavage of translation initiation factor $4 \mathrm{G}$ (eIF4G) during anti-Fas IgM-induced apoptosis does not require signalling through the p38 mitogenactivated protein (MAP) kinase. FEBS Lett 438: 41-48, 1998. 